\title{
Política de Assistência Educacional e a Taxa de Sucesso da Graduação das Universidades Públicas Federais
}

\author{
Educational Assistance Policy and the Success Rate of Graduation of the \\ Federal Public Universities \\ Política de Asistencia Educativa y la Tasa de Éxito de la Graduación de las \\ Universidades Públicas Federales \\ CRISTIANE APARECIDA DA SILVA \\ FABRICIA SILVA DA ROSA \\ ERNESTO FERNANDO RODRIGUES VICENTE \\ MARCIELE ANZILAGO
}

\begin{abstract}
Resumo: Este estudo analisou-se a eficiência das despesas com assistência educacional e sua relação com as taxas de sucessos das universidades públicas federais (UPF). A amostra compreendeu 32 UPF, nos anos de 2008 a 2014, foram analisados em dois momentos, primeiro foi aplicado o modelo de Análise Envoltória de Dados, e depois a realização da regressão de dados em painel. Esse estudo contribui para preencher lacunas de estudo em diversas disciplinas exemplos: Educação, Contabilidade Pública, Administração Pública.
\end{abstract}

Palavras-chave: Assistência Educacional. Despesas Públicas. Taxa de sucesso da Graduação.

Abstract: This study analyzed the efficiency of educational assistance expenditures and its relation to success rates of federal public universities (UPF). The sample comprised 32 UPF, in the years 2008 to 2014, that were analyzed in two moments, first the Data Envelopment Analysis model was applied, and then the panel data regression. This study contributes to filling study gaps in several disciplines, for examples: Education, Public Accounting, and Public Administration.

Keywords: educational assistance; public expenditures; graduation success rate.

Resumen: Este estudio analizó la eficiencia de los gastos con asistencia educativa y su relación con las tasas de éxitos de las universidades públicas federales (UPF). La muestra comprendió 32 UPF, en los años de 2008 a 2014, fueron analizados en dos momentos, primero fue aplicado el modelo de Análisis Envoltorio de Datos, y luego la realización de la regresión de datos en panel. Este estudio contribuye a llenar lagunas de estudio en diversas asignaturas ejemplos: Educación, Contabilidad Pública, Administración Pública.

Palabras clave: Asistencia educativa. Gastos públicos. Tasa de éxito de la graduación. 


\section{INTRODUÇÃO}

A aquisição de novos conhecimentos representa elemento crucial para se atingir um padrão de crescimento sustentável e progresso rumo a uma economia baseada no conhecimento (DI PAOLO, 2016). Nesse contexto estão circunscritas as universidades, detentoras de uma atuação essencial na produção do conhecimento e na transformação do cidadão. Dessa maneira, a avaliação da educação expressa o cumprimento das finalidades em detrimento dos meios, onde o ensino, pesquisa, extensão, infraestrutura e administração são os meios elencados para a realização da principal função da educação superior de qualidade: a construção da cidadania e a formação (graduação) de sujeitos socialmente responsáveis (DIAS SOBRINHO, 2009).

Desde o início do século XXI, vive-se um momento importante de expansão e democratização do acesso à Educação Superior pública no Brasil. Com a implantação do Programa de Reestruturação e Expansão das Universidades Federais (REUNI) em 2007, foram criadas 14 novas universidades federais e mais de 100 novos campi em todas as regiões do país. Além disso, foram diversificadas as formas de ingresso nos processos seletivos das universidades, com adoção da nota do Exame Nacional do Ensino Médio (ENEM) e da implantação das cotas etnicorraciais e sociais (SANTOS; MARAFON, 2016).

Como consequência da expansão do acesso, logo se procurou propiciar condições para aqueles alunos de famílias com vulnerabilidades socioeconômicas permanecerem e concluírem os estudos. Foi a partir desse objetivo que, em 2008, foi criado o Programa Nacional de Assistência Estudantil (PNAES), por meio da Portaria Normativa no 39, de 12 de dezembro de 2007 (BRASIL, 2007).

Os programas de assistência estudantil são, atualmente, desenvolvidos em quase todas as Instituições Federais de Ensino Superior (IFES) e incorporam ações como: alimentação, moradia, assistência à saúde, creche, acessibilidade e apoio pedagógico (SANTOS; MARAFON, 2016). Na Educação Superior, a política de assistência estudantil tem como finalidade prover os recursos necessários à transposição dos obstáculos e superação dos impedimentos ao bom desempenho acadêmico.

Dessa maneira, as políticas públicas de assistência educacional transitam em todas as áreas do conhecimento, compreendendo ações que proporcionem desde condições ideais de saúde, acesso aos instrumentais pedagógicos necessários à formação profissional nas mais diferentes áreas do conhecimento, acompanhamento às necessidades educativas especiais, até o provimento dos recursos mínimos para a sobrevivência do estudante, tais como: moradia, alimentação, transporte e recursos financeiros (ALVES, 2002). 
No entendimento de que as despesas ou gastos públicos com as políticas de assistência educacional nas universidades federais brasileiras são financiados mediante tributação, por meio da contribuição de toda a sociedade, é desejável que essas despesas sejam identificadas e analisadas. Diante desse contexto, surge a seguinte questão de pesquisa: Qual a relação entre a eficiência relativa das despesas públicas com assistência educacional e a taxa de sucesso dos cursos de graduação das universidades públicas federais? Assim, o objetivo deste estudo consiste em analisar, relativamente, a eficiência na realização das despesas com assistência educacional e sua relação com as taxas de sucesso da graduação das universidades públicas federais. Neste estudo, a eficiência relativa é definida com a comparação dos resultados dessas universidades com outras que compõem a amostra da pesquisa.

Estudos nacionais (CARVALHO, 2013; ESTRADA; RADELLI, 2014; SANTOS; MARAFON, 2016; GOMES, 2016) e internacionais (AGASISTI; MURTINU, 2013; AGASISTI; LONGOBARDI; REGOLI, 2014) que exploraram a assistência educacional nas Universidades representam contribuições à temática; porém, há, necessidade de estudos também voltados a avaliação das despesas de assistência educacionais relacionadas ao desempenho dos alunos (taxa de sucesso).

Dessa forma, infere-se que o presente estudo contribui como meio de investigar a influência das políticas públicas assistenciais, inicialmente em relação à taxa de sucesso da graduação. Portanto, esta pesquisa se mostra relevante por investigar tanto a eficiência das despesas com assistência educacional quanto a relação desta com a taxa de sucesso da graduação. Também tem contribuição prática, pois disponibiliza ferramentas úteis aos gestores para a tomada de decisão, com o propósito de subsidiar o aperfeiçoamento das políticas públicas de assistência educacionais. Nas palavras de Johnes e Johnes (2016), governos de todo o mundo fornecem subsídios para o ensino superior e, por isso, têm legítimo interesse na eficiência com que as instituições convertem entradas e saídas.

\section{REFERENCIAL TEÓRICO E HIPÓTESE DE PESQUISA}

\section{POLÍTICAS PÚBLICAS DE ASSISTÊNCIA EDUCACIONAIS}

A política de assistência aos estudantes, segundo Fonaprace (2007), consiste em um conjunto de princípios que norteiam a implantação de ações, tais como garantia ao acesso, à permanência e à conclusão do curso dos estudantes das IFES, como também respaldam o aspecto de inclusão social, formação 
ampliada, produção de conhecimento, melhoria do desempenho acadêmico e da qualidade de vida, garantindo o acesso às universidades do estudante em situação econômica desfavorável.

Na perspectiva de Meek (1991), as políticas públicas educacionais devem ser tratadas não apenas como uma diretriz de governo aceita oficialmente, mas serem vistas como resultantes da interação entre os atores-chave envolvidos em questões relacionadas com a estrutura, função e caráter de sistemas de ensino superior, o que inclui as ações e decisões do governo para implementar programas e atingir metas, bem como a interação desses atores-chave, partes interessadas ou grupos que são capazes de articular os seus interesses e ajudar, prioritariamente, as formas governamentais.

Teóricos marxistas identificam a classe social e o interesse econômico como forças motrizes na formulação de políticas nas sociedades capitalistas democráticas, enquanto teóricos 'pluralistas' afirmam que indivíduos e grupos têm uma gama de interesses. Dentro das instituições políticas, não existem 'em isolamento’ aquelas políticas a serem examinadas e que representam o impacto de forças externas, como a globalização e pressões econômicas (DOERN; PHIDD, 1983; ATKINSON, 1993).

O PNAES, implantado em 2008, teve seus objetivos atualizados e previstos no Art. $2^{\circ}$ do Decreto Presidencial no 7.234, de 19 de julho de 2010 (BRASIL, 2010), que são: democratizar as condições de permanência dos jovens na educação superior pública federal; minimizar os efeitos das desigualdades sociais e regionais na permanência, conclusão da Educação Superior; reduzir as taxas de retenção e evasão, e contribuir para a promoção da inclusão social pela educação (BRASIL, 2008).

O desenvolvimento de políticas de administração pública, voltadas principalmente ao campo da ciência política, tem contribuído conceitualmente para a compreensão da formulação de políticas. Embora conceituada e articulada de forma diferente na literatura, uma variedade de abordagens teóricas tem sido utilizada em estudos de política. A teoria da escolha pública centra-se no 'auto interesse racional' dos indivíduos dentro e fora do governo, como uma influência fundamental na formação de políticas públicas (AXELROD; DESAITRILOKEKAR; SHANAHAN; WELLEN, 2011).

Nesse contexto, enquanto cerne da política pública, o Programa de Assistência Educacional precisa percorrer as fases de acompanhamento e de avaliação, pois elas são indispensáveis, uma vez que garantem o sucesso da política e é nelas onde os resultados e impactos são verificados. Para que o monitoramento e a avaliação sejam efetivos, é necessário que os mesmos estejam previstos no desenho da política, inclusive com seus instrumentos (CONDÉ, 2012). 
Nesse segmento, Carvalho (2013) avaliou a eficácia da política de assistência educacional em relação ao alcance de equidade no rendimento acadêmico e permanência no curso, tendo constatado que o rendimento acadêmico do grupo de estudantes em situação de vulnerabilidade socioeconômica é superior ao do grupo formado pelos demais estudantes da universidade, e que os índices de evasão desses estudantes são inferiores aos dos demais.

Estrada e Radelli (2014) investigaram, por meio das entrevistas realizadas com estudantes, a política de assistência educacional na perspectiva dos estudantes assistidos por ela. Os autores enfatizaram que a pesquisa possibilitou constatar que a expansão do acesso não é somente garantir o ingresso na universidade; é necessário também gerar condições de permanência durante a vida acadêmica, pois os resultados apresentados indicaram que programas fornecedores de bolsas ou auxílios financeiros para subsidiar os gastos com moradia, alimentação e transporte são iniciativas essenciais para permanência dos estudantes na universidade.

Santos e Marafon (2016) demonstraram, em seu estudo, o desenvolvimento dos programas de assistência aos estudantes na Universidade Federal de Santa Catarina. Os resultados encontrados sugeriram que houve ampliação de todos os programas assistenciais, uma vez que o perfil socioeconômico do estudante atendido é inferior ao da totalidade dos estudantes do meio universitário, e que os desafios para assegurar a efetividade da política envolvem a insuficiência de recursos financeiros e humanos, sendo o Assistente Social o principal responsável pela permanência, a partir da complexa tarefa de selecionar os estudantes a serem atendidos.

\section{EFICIÊNCIA EDUCACIONAL}

A eficiência é, segundo Zybersztajn e Sztajn (2005), o atributo indispensável para o alcance do melhor resultado, com menor emprego de recursos ou esforços. Slomski (2005) complementa que a eficiência não deve referir-se somente à menor quantidade de recursos utilizados, mas também ao atendimento da sociedade em termos de tempestividade, oportunidade e qualidade, itens aos quais a administração pública precisaria ofertar por meio de recursos tributário arrecadados pelo governo, proporcionando o bem-estar dos cidadãos.

O cenário proposto pela literatura de controle gerencial aplicado ao setor público, com o aporte das visões gerenciais inovadoras que confrontam as formas tradicionais, as quais se baseiam em processo e são focadas nos meios e não nos fins das políticas públicas (FÉLIX SUEVO; GOMES, 2008), bem como a institucionalização emanada da Constituição Federal de 1988 (BRASIL, 
1988) introduzem o princípio da Eficiência no setor público e a necessidade de se acompanhar e fiscalizar o resultado nesse setor. Dentre os parâmetros para delimitar esse acompanhamento estão a Lei Complementar 101/00 (BRASIL, 2000), que determina a implantação de sistema de custos no setor público, a Lei Complementar 131/09 (BRASIL, 2009), a qual reforça a perspectiva da governança eletrônica no setor público, enquanto a Lei 4320/64 (BRASIL, 1964) define, ao mesmo tempo, um modelo de contabilidade pública com alcance patrimonial, financeiro e orçamentário.

Assim, o paradigma da gestão pública no Brasil necessita de mudanças urgentes em sua estrutura, a fim de buscar uma melhoria perceptível com despesas públicas de qualidade, de maneira que os recursos tributários alcancem uma efetividade relevante, a ponto de contemplar, passo a passo, ações reais, experiências replicáveis (GOMES, 2009), e no futuro, a redução da carga tributária, visto que a ideia de mais políticas públicas com menos recursos deveria ser colocada em prática.

Nesse contexto, Schwartzman (1988) relata que é necessário que a organização, nesse caso, a universidade, seja eficiente para que possa cumprir os seus compromissos com a Sociedade e o Estado. Nas palavras de Motta (1990), eficiência é um conceito relativo ao cumprimento de normas e à redução de custos, e para sua avaliação, necessita-se produzir informações com vistas a medir a utilização de insumos e seus efeitos acerca dos resultados, sendo um programa público eficiente quando consegue produzir resultados a um custo mais baixo. Para o autor, a eficiência "é essencialmente uma razão insumo/produto, receita/ despesa ou uma medida de custo/benefício" (MOTTA, 1990, p. 233).

Nesse sentido, a eficiência teria como propósito alcançar o resultado previsto gastando-se o mínimo possível; Motta (1990), porém, ressalta que cortar despesas não significa necessariamente ser mais eficiente, pois pode ser que reduzindo-se as despesas, se reduzam também os benefícios obtidos por unidade monetária, deixando o programa menos eficiente.

Concernente à educação Beuren, Moura e Kloeppel (2013) enfatizam que a análise da eficiência não deve perder de vista os objetivos finais da Educação Superior aos quais ela deve subordinar-se; por outro lado, é evidente que a utilização adequada dos recursos humanos e materiais deve ser preocupação constante em qualquer processo avaliativo, como também é algo extremamente importante e necessário, cujo objetivo principal é confrontar a adequação dessa utilização com os resultados alcançados.

Nas palavras de Agasisti, Longobardi e Regoli (2014), a eficiência educacional se refere à capacidade de realização e maximização, medida por intermédio de indicadores, tais como taxas de conclusão ou o aproveitamento 
recente de dados disponíveis nas pontuações em testes padronizados. Em outras palavras, um sistema educacional é eficiente quando, por meio de suas regras e instituições, é capaz de contribuir positivamente para estudantes realizarem seus estudos, e utilizar as políticas públicas de assistência estudantil de forma eficaz.

\section{DESPESA COM ASSISTÊNCIA EDUCACIONAL}

Com a implantação do PNAES, foi possível garantir recursos específicos às universidades para a implementação de ações de assistência estudantil, as quais passaram a ser entendidas como parte da política educacional, com objetivo de fornecer os recursos necessários para que os estudantes ultrapassem os obstáculos ao bom desempenho acadêmico (ALVES, 2002).

Assim, as despesas com assistência educacional correrão à conta das dotações orçamentárias anualmente consignadas ao Ministério da Educação (MEC), sendo tais despesas subsidiadas, nas universidades, por meio da ação orçamentária 4002, denominada Assistência ao Estudante de Ensino Superior, cuja ação é vinculada ao programa orçamentário 2032, conceituado como Educação Superior de Graduação, Pós Graduação, Ensino, Pesquisa e Extensão, devendo o Poder Executivo compatibilizar a quantidade de beneficiários com as dotações orçamentárias existentes, observados os limites estipulados na forma da legislação orçamentária e financeira (BRASIL, 2007).

Agasisti e Murtinu. (2013) relatam que a obtenção de um subsídio afeta positivamente o desempenho acadêmico. Dessa forma, os estudantes que recebem apoio financeiro, como obtenção de créditos que auxiliem na sua formação, têm menos probabilidade de abandono, principalmente no primeiro e segundo ano, e são mais propensos a formar-se dentro de quatro anos, em tempo menor quando comparados com os seus homólogos que não receberam assistência educacional. Nesse sentido, Agasisti, Longobardi e Regoli (2014) mencionam que países com maior investimento em educação no percentual da despesa pública total, e com melhores índices acerca da qualidade e quantidade dos serviços educacionais, também mostram maior proporção de estudantes resilientes, que, nas palavras de Agasisti, Longobardi e Regoli (2014), são aqueles que, apesar de seu nível socioeconômico desfavorecido, obtêm alto desempenho acadêmico.

Apesar da existência de universidades federais públicas com gratuidade do ensino no Brasil, somente com a implantação do REUNI e do PNAES é que o acesso à Educação Superior se tornou possível para os filhos da classe trabalhadora, visto que a permanência na universidade requer o atendimento de necessidades sociais objetivas desses estudantes ingressantes (transporte, alimentação, material didático, etc.) (SANTOS; MARAFON 2016). 
Nesse contexto Agasisti e Murtinu (2013) analisaram o impacto da ajuda financeira em várias dimensões do desempenho acadêmico em relação a créditos formativos obtidos após um ano e a probabilidade de abandono no primeiro e no segundo ano do tempo legal de um curso de graduação, com a duração de quatro anos. Com esses elementos, os autores encontraram um efeito estatisticamente positivo e significativo da subvenção.

Gomes (2016) buscou discutir a eficiência das despesas públicas com Educação Superior pelas universidades federais e, como resultado, o pesquisador constatou que, no triênio 2012 a 2014, oito das onze universidades avaliadas demonstraram eficiência moderada a forte com relação à aplicação do recurso público em Educação Superior, considerando as variáveis custo corrente por aluno, taxa de sucesso e IGC contínuo. Gomes (2016) utilizou como instrumentos de pesquisa entrevistas e, a partir dessas, os autores observaram fatores complicadores dessa eficiência, como pouca reflexão dos gestores acerca da temática, baixa autonomia na implantação de melhorias de gestão e ausência de corpo técnico qualificado em planejamento e controle interno.

\section{TAXAS DE SUCESSO NA GRADUAÇÃO}

A relação do estudante de nível superior com a universidade é estabelecida em sua trajetória, e se pode configurar de diversas maneiras, tanto durante quanto ao final dela, processo do qual decorrem trajetórias que podem ser classificadas como de sucesso ou insucesso (COULON, 1995). Segundo Almeida (2000), a implementação de políticas de assistência educacional torna-se uma estratégia governamental de suma importância no que se refere ao enfrentamento de problemas de acesso e permanência dos alunos nas universidades, em decorrência dos altos índices de abandono e retenção do espaço acadêmico.

$\mathrm{Na}$ acepção de Faro (2008), aproximar-se do cotidiano do estudante em situação de vulnerabilidade é essencial, pois é nele que os riscos de insucesso acadêmico se manifestam e se constituem de forma mais definitiva. A entrada na universidade exige, por parte do aluno, uma série de adaptações em níveis pessoais, sociais, financeiros e acadêmicos; portanto, entrar na universidade e nela permanecer implica adaptar-se a um novo ambiente, a uma nova cultura; consequentemente, a novas maneiras de agir e pensar.

Com o propósito de melhorar as taxas de sucesso nas universidades, o poder público tomou como iniciativa a decisão de disponibilizar políticas de acesso aos estudantes, na busca da superação das desigualdades, promovendo a equidade entre os alunos ingressantes (GRANJA, 2012). 
A Taxa de Sucesso na Graduação (TSG) nas universidades brasileiras é um indicador resultante da razão entre o número de diplomados e o número de ingressantes, ajustados pelo ano em que esses alunos ingressaram na Universidade e o tempo de permanência fixado pela Secretária de Educação Superior do Ministério da Educação (SESu/MEC) para cada curso. Esse indicador mede, de certa forma, o grau de eficiência da instituição na formação superior (BOYNARD; NOGUEIRA, 2015). Na figura 1, ilustra-se a fórmula concernente ao cálculo da TSG.

Figura 1 - Cálculos da TSG.

\begin{tabular}{|c|c|}
\hline \multirow{2}{*}{ Taxa de Sucesso na Graduação (TSG)= } & $\mathrm{N}^{0}$ de diplomados ${ }^{1}$ \\
\cline { 2 - 2 } & $\mathrm{N}^{0}$ total de alunos ingressantes ${ }^{2}$ \\
\hline
\end{tabular}

Fonte: adaptação do TCU (2006).

Para o número de diplomados (1), deve-se considerar o número de concluintes (que completaram os créditos, mesmo não tendo colado grau) dos cursos no ano letivo correspondente ao exercício, somando-se o número de concluintes nos dois semestres do ano. Para o cálculo dos números de ingressantes (2), deve ser considerado o ano do suposto ingresso dos estudantes que se graduam no exercício, com base na duração padrão prevista para cada curso. Assim, para o caso de cursos anuais com duração de 4 anos, deve ser considerado o número de ingressantes de quatro anos letivos atrás; para cursos com duração de 5 anos, devem ser considerados os ingressantes de cinco anos letivos atrás (BRASIL, 2006).

Hanushek e Woessmann (2011) ressaltam o impacto no resultado, medido pelo desempenho dos estudantes (taxa de sucesso), aspectos socioeconômicos, como a renda familiar e a escolaridade dos pais dos estudantes. Nesse sentido, Agasisti, Longobardi e Regoli (2014) analisaram os fatores que estão estatisticamente relacionados com uma maior proporção a estudantes resilientes, e os resultados encontrados sugeriram que as variações na quantidade de recursos destinados à educação poderão ter efeitos benéficos acerca do desempenho educacional (Taxa de sucesso), podendo ajudá-los a obterem oportunidades enquanto os recursos que os mesmos possuem são de certa maneira ausentes, dados seus meios desfavorecidos. Nesse contexto estabelece a seguinte hipótese de pesquisa: 
H1: Há relação positivamente significativa entre a eficiência da política de assistência educacional e o indicador de taxa de sucesso da graduação.

A "eficiência", é definida como a capacidade de produzir o máximo de serviço educacional para determinado orçamento (AGASISTI, 2017). A evolução dos sistemas de ensino superior nas últimas décadas tem sido caracterizada por uma atenção especial ao tema da "eficiência". Esse discurso está ganhando força nos momentos em que os orçamentos públicos são cada vez mais reduzidos e, consequentemente, os investimentos públicos em áreas, como o ensino superior, sofrem com um declínio acentuado dos recursos públicos a elas alocados. Ao mesmo tempo, as expectativas dos cidadãos em relação às atividades das instituições de ensino superior são ainda maiores do que no passado (HAZELKORN, 2015).

As sociedades acreditam que as instituições de ensino superior devem educar seus estudantes para serem grandes cidadãos do mundo de amanhã, um mundo que será caracterizado pela necessidade de habilidades mais sofisticadas, pela interação entre pesquisa e desenvolvimento socioeconômico e por um ciclo contínuo de inovação e transferência de conhecimento da academia para as partes interessadas externas. Dessa perspectiva, "fazer mais com menos" é agora um imperativo que caracteriza todas as atividades do setor público, incluindo o financiamento de operações e instituições de ensino superior (AGASISTI, 2017).

Nesse sentido Casado (2007) enfatiza que os procedimentos de avaliação da eficiência das universidades baseiam-se em informações concernentes aos recursos utilizados e aos resultados alcançados pela instituição sob análise e por um conjunto de instituições similares, sendo tais informações existentes nos sistemas acadêmico e contábil das universidades e, em geral, disponíveis em publicações oficiais.

\section{METODOLOGIA DA PESQUISA}

Em termos de enquadramento metodológico, este estudo se caracteriza como descritivo no que tange aos objetivos, já que tem como finalidade analisar, relativamente, a eficiência na realização das despesas com assistência educacional e sua relação com as taxas de sucesso da graduação das universidades públicas federais (Richardson, 1999). Quanto à coleta, caracteriza-se como documental. No que tange à abordagem, a pesquisa se caracteriza como quantitativa (Richardson, 1999), por empregar método estatístico.

Ressalta-se que, para alcançar o objetivo do estudo, foram realizadas duas fases: na primeira, foi realizada a análise da eficiência relativa, por intermédio do DEA, enquanto que, na segunda, foi construído um modelo estatístico com o resultado da primeira fase, em conjunto com o Indicador de TSG, e realizada uma 
regressão com dados em painel operacionalizada por meio do software Gretl. Os dados da amostra foram levantados segundo o eixo temporal de 2008 a 2014. A população compreende 62 Universidades Federais Brasileiras (UFB) e a amostra corresponde 32 UFB conforme a figura 2.

\section{Figura 2 - Mapa das Universidades Públicas Federais Brasileiras e os respectivos Estados}

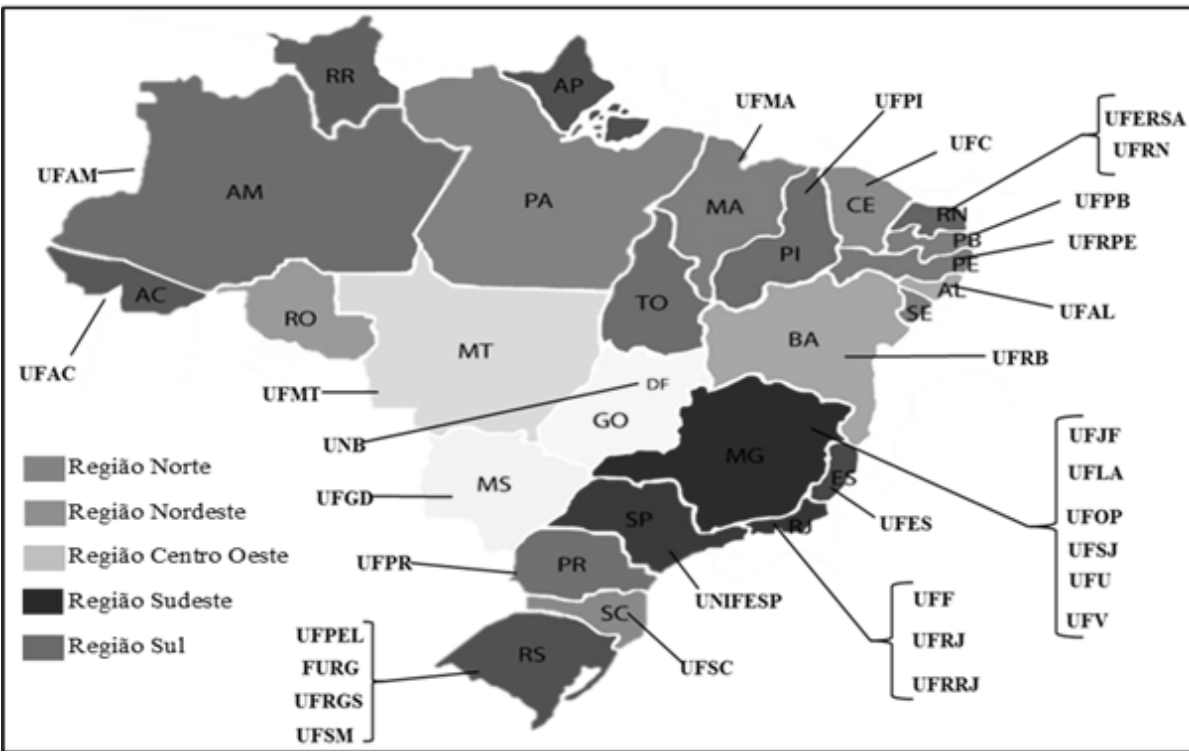

\begin{tabular}{|c|c|c|c|c|c|}
\hline Instituị ão (IIS) & Estado & Região & Instituição (IIS) & Estado & Região \\
\hline Universida de de Brasilia (UNB) & $\mathrm{DF}$ & Centro-Oeste & Univers idade F. de Juiz De Fora (UFJF) & MG & Sudeste \\
\hline Universida de F, da Grande Dourados (UF GD) & MS & Centro-Oeste & Univers idade F. de Lavras (UFLA) & MG & Sudeste \\
\hline Universidade F. de Mato Grosso (UFMCT) & $\mathrm{MT}$ & Centro-Oeste & Univers idade F. de Ouro Prato (UFOP) & MG & Sudeste \\
\hline Universidade F. de Alagoas (UFAI) & AL & Nordeste & Univers idade F. de Sảo J oảo De1Rei(UFSI) & MG & Sudeste \\
\hline Universidade F. do Recóncavo da Bahia (UFRB) & BA & Nordeste & Univers ida de F. Uberlándia (UFU) & MG & Sudeste \\
\hline Universidade F. da Bahia (UFBA) & $\mathrm{BA}$ & Nordeste & Univers idade F. de Viçosa (UFV) & MG & Sudeste \\
\hline Universida de F. do Ceara (UFC) & $C E$ & Nordeste & Univers ida de F. Fhuminense (UFF) & RJ & Sudeste \\
\hline Universida da F. do Maranhão (UFMA) & MA & Nordeste & Univers ida de F. do Rio De I ane iro (UFRJ) & RJ & Sudeste \\
\hline Universida de F. da Paaíba (UFPB) & PB & Nordeste & Univers ida de F. Rual do R io De I ane iro (UFRR.) & $R J$ & Sudeste \\
\hline Universida de F. Rural de Peman buco (UFRPE) & $\mathrm{PE}$ & Nordeste & Univers ida de F. de Sảo Paulo (UNTFESP) & SP & Sudete \\
\hline Universida de F. do P iaui (UFPD) & PI & Nordeste & Univers idade F. do Parana (UFPR) & PR & Sul \\
\hline Universida de F. Ruraldo Sem i-Árido (UFERSA) & RN & Nordeste & Univers idade F. de Pelotas (UFPEL) & RS & Sul \\
\hline Universida de F. do Rio Grande Do Norte (UFRN) & RN & Nordeste & Univers ida de F. do Rio Grande Do Sul(UFRGS) & RS & Sul \\
\hline Universida de F. do Acre (UFAC) & $A C$ & Norte & Univers ida de F. do Rio Grande (FURG) & RS & Sul \\
\hline Universida de F. do Amazonas (UFAM) & AM & Norte & Univers ida de F. de Santa Maria (UFSM) & RS & Sul \\
\hline Universida de F. do Es pirito Santo (UFES) & ES & Sudeste & Univers idade F. de Santa Catarina (UFSC) & SC & Sul \\
\hline
\end{tabular}

Fonte: Dados da pesquisa (2017). 
$\mathrm{Na}$ Figura 2, ilustram-se as Universidades com seus respectivos Estados, e também sua localização por macrorregião (Norte, Nordeste, Centro-Oeste, Sudeste e Sul).

Com o propósito de avaliar relativamente, a eficiência na realização das despesas com assistência educacional e sua relação com as taxas de sucesso da graduação das universidades públicas federais, foram estabelecidas duas fases de análise, com seis variáveis, conforme descrito na Tabela 1.

\section{Tabela 1 - Fases e variáveis da pesquisa}

\begin{tabular}{|c|c|c|c|}
\hline \multicolumn{2}{|r|}{ Variáveis } & Conceito Operacional & Autores \\
\hline \multirow{4}{*}{$1^{\text {a }}$ fase } & Dotação atualizada das despesas & $\begin{array}{l}\text { Valores monetários } \\
\text { autorizados e atualizados }\end{array}$ & $\begin{array}{l}\text { Agasisti et al., 2014 } \\
\text { Gomes } 2016\end{array}$ \\
\hline & $\begin{array}{c}\text { Despesas liquidadas com assistência } \\
\text { estudantil }\end{array}$ & $\begin{array}{c}\text { Valores destinados a } \\
\text { assistência educacional com } \\
\text { fato gerador }\end{array}$ & Agasisti et al., 2014 \\
\hline & Alunos matriculados & $\begin{array}{l}\text { Quantidade de alunos } \\
\text { matriculados nas } \\
\text { Universidades }\end{array}$ & Carvalho, 2013 \\
\hline & Benefícios concedidos & $\begin{array}{c}\text { Quantidade de benefícios } \\
\text { assistências concedidos aos } \\
\text { alunos } \\
\end{array}$ & BRASIL, 2017d \\
\hline \multirow[b]{2}{*}{$2^{\mathrm{a}}$ fase } & Indicador de taxa de sucesso da graduação & $\begin{array}{c}\text { Indicador de desempenho da } \\
\text { graduação }\end{array}$ & Gomes, 2016; \\
\hline & Indicador de eficiência relativa & $\begin{array}{l}\text { Indicador de eficiência } \\
\text { Relativa resultado da } \\
\text { primeira fase }\end{array}$ & --.-- \\
\hline
\end{tabular}

Fonte: elaboração pelos autores (2018)

Para o cálculo da eficiência relativa correspondente à primeira fase da pesquisa, utilizou-se da Análise Envoltória de Dados (DEA), por meio do software estatístico MaxDEA, proposto inicialmente por Charnes, Cooper e Rhodes (1978). O modelo adotado foi BCC (BANKER; CHARNES, COOPER,1984), também conhecido como VRS (Variable Returns to Scale), o qual utiliza uma formulação que permite a projeção de cada Decision Making Unit (DMU) ou seja para cada Unidade de Tomada de Decisão, acerca da DEA determinada pelas DMUs eficientes de tamanho compatível. Na tabela 2 apresenta-se a formação das DMUs, para a análise da primeira fase. 
Tabela 2 - Definição das unidades de análise ou conceito de eficiência

\begin{tabular}{|c|c|c|c|c|}
\hline DMU & Input 1 & Input 2 & Output 1 & Output 2 \\
\hline Universidades & DAD & DAEL & AM & BC \\
\hline
\end{tabular}

Fonte: Dados da pesquisa

Como se pode observar na tabela 2, o input 1 - Dotação Atualizada das despesas (DAD) - consiste em valores monetários autorizados e atualizados por meio de emendas parlamentares ou contingenciamentos, consignados na Lei Orçamentária Anual (LOA), para atender a uma determinada programação orçamentária (BRASIL, 2017a). O imput 2 - Despesas com assistência estudantil liquidada (DAEL) - equivale-se a valores monetários referentes à fase onde são essencialmente verificados os critérios de realização das despesas com assistência educacional, onde os valores "liquidados" remetem ao direito de receber do credor analisado, em detrimento da ocorrência do fato gerador (MACHADO; HOLANDA, 2010; CAMPELO, 2012).

O output 1 Alunos matriculados (AM) fundamenta-se nos estudantes matriculados nos cursos de graduação das universidades públicas federais, enquanto output 2 Benefícios Concedidos (BC) corresponde ao quantitativo de benefícios destinados ao apoio aos estudantes do ensino de graduação, mantendo, a critério da instituição, os restaurantes universitários, as casas de estudantes, a assistência médico-odontológica, alojamento e transporte, dentre outras iniciativas típicas de assistência social ao educando, cuja concessão seja pertinente sob o aspecto legal, e contribua para o bom desempenho do aluno na universidade (BRASIL, 2017c; BRASIL, 2017a).

As variáveis DAD E DAEL foram coletadas por meio do Sistema de Planejamento e Orçamento do Governo Federal (SIOPE). As variáveis AM e BC foram coletadas por meio do Relatório de Gestão das Universidades Federais, disponíveis no site do Tribunal de Contas da União (TCU), e no Orçamento Federal, disponibilizado no site do Ministério do Planejamento Orçamento e Gestão (MPOG).

Para análise da segunda fase, foram coletados os Indicadores das Taxas de Sucesso (TSG), disponível no Relatório de Gestão das Universidades, extraído do site do TCU, e realizou-a regressão de dados em painel com os indicadores de eficiência relativa (encontrado na primeira fase) e os indicadores da TSG utilizando o software Gretl. Ressalta-se que tanto os dados da primeira fase quanto os dados da segunda foram coletados nos períodos de maio a julho de 2017. 


\section{ANÁLISE E DISCUSSÃO DOS RESULTADOS}

Inicialmente, apresenta-se o cálculo da eficiência na execução das despesas públicas com assistência estudantil das universidades federais brasileiras, seguido dos indicadores da Taxa de Sucesso da Graduação das Universidades Brasileiras, e por último, os resultados obtidos da técnica de dados em painel e discussões dos resultados. Na Tabela 3, apresentam-se os scores de eficiência de cada universidade.

\section{Tabela 3 - Scores de Eficiência}

\begin{tabular}{|c|c|c|c|c|c|c|c|}
\hline Universidades & 2008 & 2009 & 2010 & 2011 & 2012 & 2013 & 2014 \\
\hline UFAC & 0,12395 & 0,577723 & 0,718531 & 1 & 0,664221 & 0,43693 & 0,432722 \\
\hline UFAL & 0,157751 & 0,513157 & 0,7116 & 0,617649 & 0,71121 & 0,690004 & 0,695996 \\
\hline UFAM & 0,626163 & 1 & 1 & 0,549017 & 0,536573 & 0,49329 & 0,489799 \\
\hline UFRB & 0,111462 & 0,391289 & 1 & 0,68149 & 0,50557 & 0,327544 & 0,388094 \\
\hline UFBA & 0,256761 & 0,720991 & 0,807513 & 1 & 0,874568 & 1 & 0,850476 \\
\hline UFC & 0,166506 & 0,758098 & 0,693492 & 0,546682 & 0,637118 & 1 & 0,788988 \\
\hline UNB & 1 & 0,832961 & 1 & 0,917532 & 0,658722 & 0,857159 & 1 \\
\hline UFES & 0,234471 & 0,626745 & 0,57952 & 0,558725 & 0,601061 & 0,49056 & 0,514843 \\
\hline UFMA & 0,347972 & 0,764484 & 0,545277 & 0,786765 & 0,796263 & 0,984831 & 0,897334 \\
\hline UFJF & 0,10898 & 0,385042 & 0,443426 & 0,440463 & 1 & 0,588382 & 0,865953 \\
\hline UFLA & 0,362995 & 0,602121 & 0,789655 & 0,647673 & 1 & 1 & 1 \\
\hline UFOP & 0,080722 & 0,325484 & 0,692635 & 0,569035 & 0,457547 & 0,429452 & 0,577656 \\
\hline UFSJ & 0,060433 & 1 & 1 & 0,695209 & 1 & 1 & 1 \\
\hline UFU & 0,135542 & 0,799995 & 0,828996 & 0,746222 & 0,650482 & 0,763242 & 1 \\
\hline UFV & 0,134105 & 0,354412 & 0,559623 & 0,563803 & 0,623915 & 0,567924 & 0,90374 \\
\hline UFGD & 0,108234 & 0,909433 & 1 & 1 & 1 & 1 & 0,465282 \\
\hline UFMT & 1 & 0,571847 & 0,45405 & 0,678069 & 0,832049 & 0,794823 & 0,699738 \\
\hline UFPB & 0,404579 & 0,625347 & 1 & 1 & 0,694537 & 1 & 1 \\
\hline UFRPE & 0,05467 & 0,709241 & 0,622523 & 0,632875 & 0,503925 & 0,473729 & 1 \\
\hline UFPI & 0,126621 & 0,655505 & 0,670642 & 0,714349 & 0,497317 & 0,595915 & 0,59501 \\
\hline UFPR & 0,378908 & 0,64735 & 0,66334 & 0,724627 & 0,670747 & 0,772246 & 0,732587 \\
\hline UFF & 0,244204 & 1 & 1 & 1 & 1 & 1 & 1 \\
\hline UFRJ & 0,261334 & 1 & 0,940111 & 1 & 1 & 0,856643 & 0,879631 \\
\hline UFRRJ & 0,090707 & 0,475037 & 0,391701 & 0,515831 & 0,474106 & 0,481297 & 0,56413 \\
\hline UFERSA & 1 & 1 & 1 & 1 & 1 & 0,57256 & 0,586255 \\
\hline UFRN & 0,136304 & 0,690228 & 0,664131 & 0,715932 & 0,909727 & 0,930789 & 0,992137 \\
\hline UFPEL & 0,119516 & 0,663697 & 0,496212 & 0,620816 & 0,801333 & 0,843664 & 0,742073 \\
\hline UFRGS & 0,182815 & 0,697744 & 0,599178 & 0,66533 & 0,573531 & 0,708266 & 0,69992 \\
\hline
\end{tabular}


Tabela 3 - Scores de Eficiência

\begin{tabular}{|c|c|c|c|c|c|c|c|}
\hline Universidades & $\mathbf{2 0 0 8}$ & $\mathbf{2 0 0 9}$ & $\mathbf{2 0 1 0}$ & $\mathbf{2 0 1 1}$ & $\mathbf{2 0 1 2}$ & $\mathbf{2 0 1 3}$ & $\mathbf{2 0 1 4}$ \\
\hline FURG & 0,121814 & 0,604496 & 0,626534 & 0,525639 & 0,487801 & 0,449519 & 0,670915 \\
\hline UFSM & 1 & 0,39863 & 0,482226 & 0,875924 & 0,903171 & 0,828671 & 0,73951 \\
\hline UFSC & 0,17702 & 0,769675 & 0,717247 & 1 & 0,770281 & 1 & 0,868777 \\
\hline UNIFESP & 0,052765 & 1 & 0,675355 & 0,747414 & 1 & 1 & 0,999348 \\
\hline
\end{tabular}

Fonte: Dados da pesquisa.

Para os índices de eficiência, deve se levar em consideração a escala entre 0 e 1: quanto mais próximo de 1 mais eficiente será a universidade; quando o indicador for 1 indicará que a universidade foi totalmente eficiente no período, quanto mais próximo de zero menos eficiente a universidade foi. Dessa forma, o conceito de eficiência refere-se, nessa perspectiva, ao que foi produzido em relação ao que poderia ter sido produzido utilizando-se os mesmos recursos (MELO; MEZA; GOMES; NETO, 2005), ou seja, a eficiência é vista como a habilidade para produzir outputs ou serviços com um nível mínimo de recursos necessários (AVKIRAN, 2001).

Dessa forma, conforme se observa nos dados apresentados na Tabela 3, percebe-se que a UFF foi a universidade mais eficiente ao longo do período analisado, obtendo o índice 1, que indica eficiência total entre os anos de 2009 e 2014, ou seja, por seis anos consecutivos. Dessa forma, depreende-se que essa universidade produziu mais serviços com um nível mínimo de recursos necessários. A Universidade de Brasília (UNB) foi totalmente eficiente nos anos de 2008, 2010 e 2014. A UFSJ foi eficiente nos anos de 2009 a 2014 consecutivamente, seguidos pela UFGD, sendo eficiente de 2010 a 2013; UFLA, de 2012 a 2014; UFPB nos anos de 2010, 2011, 2013 e 2014; UFERSA nos anos de 2008 a 2012.

As demais universidades estudadas atingiram o nível 1 (um) de totalmente eficiente somente em 1 ou 2 anos; nos demais anos, elas não conseguiram produzir outputs ou serviços com um nível mínimo de recursos necessários. A universidade menos eficiente, ou seja, que não atingiu o coeficiente máximo de eficiência de 1 (um) em nenhum dos períodos analisados foram a UFRRJ, seguida pela UFOP e UFAL. As Universidades FURG, UFRGS, UFPEL, UFRN, UFRRJ, UFPR, UFV, UFOP, UFMA, UFES e UFAL não foram eficientes em nenhum ano ao longo do período analisado, pois não conseguiram produzir serviços com um nível mínimo de recursos. Na Tabela 4, apresentam-se os indicadores da Taxa de Sucesso da Graduação das Universidades Brasileiras. 
Tabela 4 - Indicadores da taxa de Sucesso da Graduação

\begin{tabular}{|c|c|c|c|c|c|c|c|}
\hline Universidades & 2008 & 2009 & 2010 & 2011 & 2012 & 2013 & 2014 \\
\hline UFAC & 0,53 & 0,31 & 0,30 & 0,55 & 0,39 & 0,41 & 0,43 \\
\hline UFAL & 0,69 & 0,40 & 0,28 & 0,34 & 0,41 & 0,40 & 0,46 \\
\hline UFAM & 0,69 & 0,58 & 0,45 & 0,35 & 0,38 & 0,36 & 0,35 \\
\hline UFRB & 0,56 & 0,68 & 0,45 & 0,50 & 0,61 & 0,36 & 0,90 \\
\hline UFBA & 0,70 & 0,67 & 0,71 & 0,65 & 0,51 & 0,49 & 0,43 \\
\hline UFC & 0,01 & 0,73 & 0,68 & 0,69 & 0,67 & 0,57 & 0,60 \\
\hline UNB & 0,71 & 0,75 & 0,55 & 0,36 & 0,59 & 0,48 & 0,40 \\
\hline UFES & 0,62 & 0,61 & 0,65 & 0,58 & 0,64 & 0,53 & 0,51 \\
\hline UFMA & 0,89 & 0,94 & 0,51 & 0,41 & 0,53 & 0,82 & 0,40 \\
\hline UFJF & 0,72 & 0,77 & 0,82 & 0,72 & 0,79 & 0,7 & 0,61 \\
\hline UFLA & 0,72 & 0,85 & 0,65 & 0,61 & 0,55 & 0,55 & 0,37 \\
\hline UFOP & 0,76 & 0,81 & 0,81 & 0,67 & 0,63 & 0,52 & 0,50 \\
\hline UFSJ & 0,90 & 0,99 & 0,77 & 0,25 & 0,59 & 0,42 & 0,50 \\
\hline UFU & 0,74 & 0,69 & 0,69 & 0,65 & 0,65 & 0,57 & 0,54 \\
\hline UFV & 0,61 & 0,62 & 0,67 & 0,66 & 0,56 & 0,50 & 0,50 \\
\hline UFGD & 0,63 & 0,35 & 0,51 & 0,51 & 0,62 & 0,56 & 0,38 \\
\hline UFMT & 0,47 & 0,52 & 0,53 & 0,50 & 0,52 & 0,64 & 0,39 \\
\hline UFPB & 0,63 & 0,59 & 0,47 & 0,39 & 0,50 & 0,40 & 0,41 \\
\hline UFRPE & 0,59 & 0,59 & 0,58 & 0,51 & 0,50 & 0,43 & 0,34 \\
\hline UFPI & 0,58 & 0,56 & 0,52 & 0,56 & 0,57 & 0,61 & 0,56 \\
\hline UFPR & 0,81 & 0,79 & 0,77 & 0,64 & 0,63 & 0,49 & 0,62 \\
\hline UFF & 0,77 & 0,73 & 0,74 & 0,64 & 0,59 & 0,56 & 0,44 \\
\hline UFRJ & 0,51 & 0,50 & 0,90 & 0,75 & 1,00 & 0,79 & 0,80 \\
\hline UFRRJ & 0,61 & 0,60 & 0,44 & 0,41 & 0,27 & 0,26 & 0,36 \\
\hline UFERSA & 0,65 & 0,67 & 0,48 & 0,82 & 0,36 & 0,40 & 0,32 \\
\hline UFRN & 0,55 & 0,38 & 0,67 & 0,70 & 0,52 & 0,46 & 0,60 \\
\hline UFPEL & 0,72 & 0,61 & 0,65 & 0,55 & 0,36 & 0,32 & 0,41 \\
\hline UFRGS & 0,56 & 0,60 & 0,63 & 0,63 & 0,63 & 0,63 & 0,57 \\
\hline FURG & 0,62 & 0,58 & 0,55 & 0,50 & 0,51 & 0,51 & 0,35 \\
\hline UFSM & 0,67 & 0,55 & 0,54 & 0,63 & 0,81 & 0,54 & 0,55 \\
\hline UFSC & 0,62 & 0,65 & 0,72 & 0,72 & 0,63 & 0,56 & 0,51 \\
\hline UNIFESP & 0,93 & 0,77 & 0,45 & 0,46 & 0,46 & 0,14 & 0,22 \\
\hline
\end{tabular}

Fonte: Dados da pesquisa.

Com base nos dados apresentados na Tabela 4, quanto mais próximo de 1, maior a taxa de sucesso na graduação e quanto mais próximo de 0 (zero) menor a taxa de sucesso da graduação. Assim, observa-se que, os indicadores de taxa de 
sucesso na graduação, calculados pela média dos anos estudados têm maiores índices (mais próximos de 1) nas instituições: UFRJ, UFJF, UFPR, UFOP, UFU, UFMA, UFF, UFSJ, UFSC e UFLA, enquanto as universidades com menores índices de taxa de sucesso na graduação (mais próximos ao índice 0) são: UFAC, UFRRJ, UFAL, UFAM, UFPB e UNIFESP. Dessa forma, percebe-se, por meio dos dados da Tabela 3 e 4, que as universidades eficientes nem sempre são as melhores com relação à taxa de sucesso nos cursos de graduação e vice-versa. Isso significa que nem sempre as universidades que produzem serviços com um nível mínimo de recursos necessários terão sucesso na taxa de graduação dos estudantes, ou seja, essas universidades nem sempre terão mais estudantes diplomados que as que não são eficientes na distribuição de seus recursos.

Para alcançar os objetivos desta pesquisa bem como a análise das hipóteses propostas, esperava-se que o índice da taxa de sucesso possuísse uma relação positiva e significativa com as proxies de eficiência das despesas com a assistência estudantil, mas conforme os resultados apresentados nas Tabelas anteriores não foram encontrados essa relação. Dessa maneira, deve-se estabelecer primeiramente qual é o painel mais indicado para análise dos dados. Na Tabela 5, apresentam-se os resultados dos testes.

\section{Tabela 5 - Resultados da regressão em painel}

\begin{tabular}{|c|c|c|}
\hline Testes & Hipóteses & Resultado \\
\hline F de Chow & \multirow{3}{*}{$\begin{array}{l}\text { H0 - Os coeficientes de eficiência } \\
\text { das despesas com assistência } \\
\text { educacional têm relação positiva e } \\
\text { significativa com índice da taxa de } \\
\text { sucesso da graduação } \\
\text { H1 - Os coeficientes de eficiência } \\
\text { das despesas com assistência } \\
\text { educacional têm relação negativa e } \\
\text { significativa com índice da taxa de } \\
\text { sucesso da graduação. }\end{array}$} & $\begin{array}{c}F=2,771 \\
\text { Sig. } X 2=0,000\end{array}$ \\
\hline LM de Breusch-Pagan & & $\begin{array}{c}X 2=23,52 \\
\text { Sig. X2 }=0,000\end{array}$ \\
\hline Teste de Hausman & & $\begin{aligned} X 2 & =3,47 \\
\text { Sig. } X 2 & =0,062\end{aligned}$ \\
\hline
\end{tabular}

Fonte: Dados da pesquisa

Com base na Tabela 5, constata-se que o modelo para da $\neg$ dos em painel com efeitos fixos foi o mais indicado para a análise dos dados de eficiência das despesas públicas com assistência educacional. O modelo de efeitos fixos é a opção adequada devido ao melhor efeito de explicação do modelo testado em relação aos demais modelos, visto que captura o efeito de todas as variáveis que não mudam ao longo do tempo. Além disso, nas validações dos modelos, verificou-se, por meio dos testes de Wooldridge para autocorrelação e Likelihoodratio para heteroscedasticidade, que o modelo não apresenta problemas de 
autocorrelação, indicando que as variáveis não apresentam correlação ao longo do tempo e heteroscedasticidade indicando que não existe uma relação linear entra as variáveis explicativas com a variável resposta.

$\mathrm{Na}$ Tabela 6, apresentam-se os resultados das regressões com dados em painel para os três modelos do estudo.

Tabela 6 - Modelo de dados em painel

\begin{tabular}{|c|c|c|c|c|}
\hline Variável & Coeficiente & Erro Padrão & Estatística T & Sig. \\
\hline Constante & 0,644 & 0,030 & 21,804 & 0,000 \\
\hline IEDA & $-0,110$ & 0,042 & $-2,648$ & 0,009 \\
\cline { 1 - 4 } R2 & \multicolumn{5}{|r|}{0,315} \\
R2 Ajustado & \multicolumn{5}{r}{0,201} \\
\hline
\end{tabular}

Fonte: Dados da pesquisa.

No modelo apresentado na Tabela 6, verifica-se que os coeficientes de eficiência das despesas com assistência educacional têm relação negativa e significativa em relação ao índice da taxa de sucesso, e por conta disso, rejeita-se H1: a qual previa relação positiva e significativa entre eficiência das despesas com assistência educacional e o índice da taxa de sucesso da graduação. Percebe-se que o resultado da regressão foi ao encontro da análise dos indicadores calculados por meio da análise envoltória de dados nas Tabelas 3 e 4, a qual não encontrou relação entre a eficiência das universidades e a taxa de sucesso da graduação dessas universidades, fato esse que pode ser devido às regiões onde estão localizadas as universidades federais.

Os resultados vão ao encontro parcial dos resultados do estudo de Gomes (2016), que buscou discutir a eficiência do gasto público em Educação Superior das universidades federais e, com os resultados obtidos, o autor constatou que, no triênio estudado, oito das onze universidades avaliadas demonstraram eficiência moderada a forte com relação à aplicação do recurso público em Educação Superior, consideradas as variáveis custo corrente por aluno, taxa de sucesso e Índice Geral de Cursos Avaliados na Instituição (IGC contínuo). No presente estudo foram utilizadas, com o propósito de avaliação da eficiência relativa correspondente à primeira fase da pesquisa, as variáveis concernentes a: i) despesas com assistência estudantil; ii) quantidade de alunos matriculados e quantidade de benefícios concedidos. Nos anos de 2008; 2009; 2010; 2011; 2012; 2013 e 2014 foram eficientes, respectivamente: três, cinco, oito, sete, sete, sete e sete das trinta e duas universidades avaliadas nos setes anos pesquisadas. 
No entanto, as informações apontam, em relação à segunda fase da pesquisa, que consistiu em verificar a relação entre os indicadores de eficiência dos gastos com assistência educacional e a variável TSG, achados contrários aos resultados de Agasisti e Murtinu (2013) e Carvalho (2013), que analisaram o impacto da ajuda financeira em várias dimensões do desempenho acadêmico. Os primeiros autores encontraram um efeito estatisticamente positivo e significativo da subvenção, enquanto Carvalho (2013) avaliou a eficácia da política com relação ao alcance de equidade no rendimento acadêmico e permanência no curso.

Constatou-se que o rendimento acadêmico do grupo de estudantes em situação de vulnerabilidade socioeconômica é superior ao do grupo formado pelos demais estudantes da universidade, e que os índices de evasão desses estudantes são inferiores aos dos demais.

Os achados deste estudo não encontram similaridade aos resultados de Agasisti et al. (2014), embora com escopo distinto, mas com certa harmonização. Os autores usaram variáveis distintas, como: a) produto interno bruto per capita; $b$ ) participação de alunos com origem de imigrações; c) proporção de estudantes cujo pais tem emprego em tempo integral; e usaram, também, variáveis semelhantes: a) gastos públicos investidos no setor educacional; b) proporção de estudantes resilientes a nível nacional). Os resultados revelaram direções opostas, ou seja, Agasisti et al. (2014), analisaram os fatores que estão estatisticamente relacionados com maior proporção a estudantes resilientes, e os resultados obtidos sugeriram que a variação na quantidade de recursos destinados à educação pode ter um efeito benéfico acerca do desempenho educacional, e pode ajudá-los a obter essas oportunidades e recursos que são de certa maneira ausentes, dado o seu meio desfavorecido.

Neste estudo, conclui-se que a análise da eficiência dos gastos com a assistência educacional não teve relação com os indicadores da Taxa de sucesso dos alunos de graduação das universidades selecionadas na amostra, não refletindo os indicadores de eficiência dos gastos no aumento ou redução do Indicador da TSG.

Contudo, tais resultados devem ser interpretados com cautela, em função do acesso temporal e de distintas localidades de algumas instituições, recomendando que futuras pesquisas investiguem outros elementos que possam explicar as diferenças encontradas, como também se torna relevante estudo com mais variáveis para perceber o real impacto das despesas com políticas públicas de Assistência educacional em relação ao Desempenho (Taxa de Sucesso da Graduação). Assim, destaca-se a necessidade de novas pesquisas e mais discussões acerca das despesas com Assistência Educacional nas universidades públicas federais brasileiras. 


\section{CONCLUSÃO E OPORTUNIDADES PARA FUTURAS PESQUISAS}

O estudo consistiu em analisar a eficiência relativa na realização das despesas com assistência educacional e sua relação com as taxas de sucesso da graduação das universidades públicas federais no período de 2008 a 2014, utilizando a análise envoltória de dados (DEA) e a análise de dados em painel base fixa, por intermédio do software Gretl.

Alicerçada nos dados analisados, conclui-se que não há uma relação entre o índice de taxa de sucesso com as proxies de eficiência das despesas de assistência educacional. Em virtude dos fatos mencionados da análise dos resultados supramencionada, as despesas com assistência educacional não cumpriram todos os objetivos do PNAES, dentre eles, o de minimizar os efeitos da permanência e conclusão da Educação Superior, ou seja, reduzir as taxas de retenção e evasão, consequentemente, e principalmente, a ampliação da Taxa de Sucesso. Tendo em vista os aspectos observados, concluiu-se que a eficiência das despesas com Políticas Públicas de Assistência Educacional não teve relação e, consequentemente, não influenciou a Taxa de Sucesso da Graduação.

Esse estudo contribui para diversas áreas, a saber: Administração Pública, Contabilidade Pública, Contabilidade Gerencial e Educação. Assim, a análise da relação entre a eficiência das despesas com assistência estudantil e a Taxa de sucesso da Graduação é importante para determinar se os investimentos na assistência educacional influenciam os resultados acerca do desempenho da graduação nas universidades públicas federais. Entender como esses investimentos se relacionam com o propósito de auxiliar efetivamente o desempenho acadêmico dos cursos de graduação é a chave para o fomento das políticas educacionais.

Além das percepções e implicações fornecidas por esta pesquisa, ou seja, de que o indicador de eficiência não teve relação com o indicador da Taxa de Sucesso da Graduação, este estudo apresenta limitações que se devem às decisões dos pesquisadores acerca do delineamento metodológico. Cabe observar que poucas são as informações disponibilizadas pelas universidades acerca das especificidades dos benefícios concedidos capazes de contribuir na análise da eficiência das despesas com a política de assistência educacional. Acerca das características dos estudantes contemplados com os benefícios, como informações dos aspectos familiares, Hanushek e Woessmann (2011) destacam que esses elementos podem impactar o resultado medido pelo desempenho dos estudantes. Aspectos socioeconômicos, como a renda familiar e a escolaridades dos pais dos estudantes, também têm a possibilidade de ser refletidas. No entanto, essas limitações possibilitam a oportunidade de pesquisas futuras. 
Recomenda-separa futuras pesquisas, além dasvariáveis supramencionadas, a investigação de outros elementos que expliquem os baixos índices de eficiência dos gastos públicos com assistência educacional nas universidades. As pesquisas futuras poderiam analisar a relação dos mecanismos de controle interno dos gastos públicos com assistência educacional nas universidades, pois a pesquisa documentada neste trabalho mostra que um dos objetivos da política pública de assistência educacional não teve cumprida sua meta, que seria uma correlação positiva entre eficiência das Universidades e o Indicador da Taxa de Sucesso da Graduação.

Há espaço também para explorar a composição do corpo discente, as disparidades de gênero que ocorrem entre as Universidades em relação aos gastos com assistência educacional e a taxa de sucesso. Em relação à análise da taxa de sucesso, a alteração do coeficiente poderá ser explicada também por outros fatores, podendo analisar aquelas universidades que têm, no seu elenco, um percentual maior de cursos noturnos em relação àquelas com mais cursos diurnos.

Além disso, as pesquisas futuras poderiam examinar os fatores que influenciam as taxas de sucesso em universidades mais antigas, como também poderão fazer uma conexão acerca da influência da gestão universitária nas mesmas. Finalmente, em relação tanto à Eficiência das despesas quanto à Taxa de sucesso da graduação, as novas pesquisas poderão ser mais profundas acerca dos processos culturais e estruturas intrínsecas e informais, que produzem e reproduzem desigualdades regionais, onde é possível também que haja interferências de políticas internas, como o planejamento e execução das políticas públicas educacionais que interfiram na eficiência das mesmas.

\section{REFERENCIAS}

AGASISTI, T. Management of Higher Education Institutions and the Evaluation of their Efficiency and Performance, jun. 2017.

. "Does public spending improve educational resilience? A longitudinal analysis of OECD-PISA data", SIEP - Italian Society of Public Economics. Working Paper n. 669, 2014.

AGASISTI, T; MURTINU, S. Are we Wasting Public Money? No! The Effects of Grants on Italian University Students' Performances. Institut d'Economia de Barcelona. Working Paper n. 33, outubro de 2013. 
ALMEIDA, N. L. T. Educação pública e Serviço Social. Serviço Social \& Sociedade em Revista, n. 63, p. 62-75, São Paulo: Editora Cortez, 2000.

ALVES, J. M. A. Assistência estudantil no âmbito da política de educação superior pública. Serviço Social em Revista, v. 5, n. 1. 2002. http://www.uel.br/revistas/ ssrevista/c-v5n1.htm, acessado em: 08 agosto, 2017.

ATKINSON, M. M. Governing Canada: Institutions and Public Policy, Toronto: Harcourt Brace Jovanovich, 1993.

AVKIRAN, N. Investigating technical and scale efficiency of Australian Universities through data envelopment analysis. Socio Economic Planning Sciences, v. 35, p. 57-80, March 2001.

AXELROD, P.; DESAI-TRILOKEKAR, R.; SHANAHAN, T.; WELLEN, R. People, Processes, and Policy-Making in Canadian Post-Secondary Education, 1990-2000. Higher Education Policy, v. 24, p. 143-166, June 2011.

BANKER, R. D.; CHARNES, A.; COOPER, W. W. Some Models for Estimating Technical and Scale Inefficiencies in Data Envelopment Analysis. Management Science, v. 30, n. 9, 1078-1092, set, 1984.

BEUREN, I. M.; MOURA, G. D. M.; KLOEPPEL, N. R. Práticas de governança eletrônica e eficiência na utilização das receitas: uma análise nos estados brasileiros. Rev. Adm. Pública, v. 47, n. 2, p. 421-441, 2013.

BOYNARD, K. M. S.; NOGUEIRA, J. M. Indicadores de gestão em conflito com indicadores de qualidade? Lições econômicas para a gestão universitária. Revista GUAL, v. 8, n. 4, p. 237-258, 2015.

BRASIL, Tribunal de Contas da União. Contas e relatórios de Gestão. http:/ / portal.tcu.gov.br/contas/contas-e-relatorios-de-gestao. Acesso em: 05 agos. 2017.

MINISTÉRIO DO PLANEJAMENTO ORÇAMENTO E GESTÃO. Secretaria de Orçamento Federal. Orçamentos Anuais. http://www. orcamentofederal.gov.br/clientes/portalsof/portalsof/orcamentos-anuais. 2015 Acesso em: 10 jul. 2017a 
Sistema Integrado de Planejamento e Orçamento. Execução

Orçamentária. https://www1.siop.planejamento.gov.br/QvAJAXZfc/opendoc. htm?document=IAS\%2FExecucao_Orcamentaria.qvw\&host=QVS $\% 40$ pqlk04 \&anonymous=true. Acesso em: 10 julho, 2017b

. MINISTÉRIO DA EDUCAÇÃO. Lista de Universidades.: < http:// portal.mec.gov.br/projeto-escola-que-protege/lista-de-universidades, Acesso em: 12 abril, de 2017 c.

Decreto Presidencial ${ }^{\circ}$ 7.234, de 19 de julho de 2010. Dispõe sobre o Programa Nacional de Assistência Estudantil - PNAES. < http://www.planalto. gov.br/ccivil_03/_ato2007-2010/2010/decreto/d7234.htm. Acesso em: 10 ago, 2017d;

. Lei Complementar n ${ }^{\mathbf{0}}$ 131, de 27 de maio de 2009 http:/ /www.planalto. gov.br/ccivil_03/leis/lcp/lcp131.htm. Acesso em: 10 ago. 2017

. Portaria Normativa $\mathbf{n}^{\mathbf{0}}$ 39, de 12 de dezembro de 2007. Institui o Programa Nacional de Assistência Estudantil - PNAES, 2007. http://portal.mec. gov.br/seed/arquivos/pdf/ead/port_40.pdf. Acesso em: 05 de ago. 2017.

- TCU-TRIBUNAL DE CONTAS DA UNIÃO. Orientações para o cálculo dos indicadores de gestão: decisão plenária n 408/2002, 2006.

. Lei Complementar $\mathbf{n}^{\mathbf{0}}$ 101, de 4 de maio de 2000. http:/ /www.planalto. gov.br/ccivil_03/leis/LCP/Lcp101.htm. Acesso em: 10 ago. 2017.

Constituição da República Federativa do Brasil. Brasília, DF: Senado Federal, 1988. https://www2.senado.leg.br/bdsf/bitstream/handle/ id/518231/CF88_Livro_EC91_2016.pdf. Acesso em: 05 de ago, 2017.

Lei $\mathbf{n}^{\circ}$ 4.320, de 17 de março de 1964. http://www.planalto.gov.br/ ccivil_03/leis/L4320.htm. Acesso em: 10 ago. 2017.

CAMPELO, M. Contribuição ao estudo de um modelo conceitual de excelência em Gestão Pública. In: Machado, N. et al. GBRSP - Gestão baseada em resultado no setor público: uma abordagem para implementação em prefeituras, câmaras municipais, autarquias, fundações e unidades organizacionais. São Paulo: Atlas, p. 211-240, 2012. 
CARvalHo, S. C. S. Avaliação da eficácia da Política Pública de Assistência Estudantil na Universidade Federal de Lavras. 2013. Dissertação (Mestrado em Administração Pública) UFLA, Lavras, 130p. 2013.

CASADO, F. L. Análise Envoltória de Dados: conceitos, metodologia e estudo da arte na Educação Superior. Revista Sociais e Humanas, v. 20, nº 1, p. 59-71, jan./jun. 2007

CHARNES, A., COOPER, W. W.; RHODES, E. Measuring the Efficiency of Decision Making Units. European Journal of Operational Research, v. 2, no 6, p. 429-444, november 1978.

CONDÉ, E. S. Abrindo a Caixa: dimensões e desafios na análise de políticas públicas. Revista Pesquisa e Debate em Educação, v.2, nº 2, p.78-100, 2012.

COULON, A. Etometodologia e Educação. Rio de Janeiro: Vozes, 1995.

DI PAOLO, A. (Endogenous) occupational choices and job satisfaction among recent Spanish $\mathrm{PhD}$ recipients. International Journal of Manpower. v. 37, $\mathrm{n}^{\circ}$ 3, p. 511-535, 2016.

DIAS SOBRINHO, J. Avaliação Institucional para Re-institucionalizar a Educação como Bem .Público. Revista de Estudos em Educação, v. 11, n. 2, p. 17-25, 2009.

DOERn, B. G., PHIDD, R. W. Canadian Public Policy: Ideas, Structure, Process, Toronto: Metheun, 1983.

ESTRADA, A. A., RADAELLI, A. A política de assistência estudantil em uma universidade pública: a perspectiva estudantil. Revista online de Política e Gestão Educacional, nª 16, 2014.

FARO, A. Os desafios da assistência estudantil como política de inclusão. $\quad h t t p: / / w w w . u f p a \cdot b r /$ fonaprace/índex.php?option=comcontent\&view $=$ article $\&=54$ :os- desafios-da-assistênciaestudantil-como-politicade-inclusao\&catid=1: ultimasnoticias\&itemid=50, 2008. Acesso em: 5 ago. 2017. 
FÉLIX SUEVO, C. L.; GOMES, J.S. Sistema de contabilidade gerencial aplicada à administração pública: um estudo de caso na COMLURB. Revista de Informação Contábil (UFPE), v. 2, p. 1-18, abr-jun/2008.

FÓRUM NACIONAL DE PRÓ-REITORES DE ASSUNTOS ESTUDANTIS E COMUNITÁRIOS. Plano Nacional de Assistência Estudantil. Brasília: FONAPRACE, 2007. http://www.ufpa.br/fonaprace, Acesso em 05 fev. 2018

GOMES, E. G. M. Gestão por Resultados e eficiência na Administração Pública: uma análise à luz da experiência de Minas Gerais. 2009. 187 p. (Tese de Doutorado em Administração Pública e Governo) - Fundação Getúlio Vargas, São Paulo, 2009.

GOMES, J. A. V. Eficiência do gasto público em educação superior: um estudo sobre as universidades federais do Estado de Minas Gerais. 2016. 75p. Dissertação de mestrado: Programa de Pós-Graduação em Administração Pública em Rede Nacional (PROFIAP). Universidade Federal de Viçosa: Campus Florestal. Rio Paranaíba - MG, 2016.

GRANJA, V. A. V. Tendências de sucesso no percurso acadêmico do alunado na UFRN. 2012. 124 f. Dissertação (Mestrado em Educação) - Universidade Federal do Rio Grande do Norte, Natal, 2012.

HANUSHEK E. A.; WOESSMANN, L. The Economics of International differences in Educational Achievement. Handbook of the Economics of Education, 3, p. 89-200, Elsevier, 2011.

HAZELKORN, E. Rankings and the reshaping of higher education: The battle for world-class excellence. 2nd edn. London: Palgrave Macmillan. 304 pp, 2015.

JOHNES, G.; JOHNES, J. Costs, efficiency, and economies of scale and scope in the English higher education sector. Oxford Review of Economic Policy, v. 32 no 4, 596-614, january 2016.

MACHADO, M. P. O pacto neodesenvolvimentista e as políticas de expansão da educação superior no Brasil de 2003 a 2013. 2013. 218f. Tese (Doutorado em Educação) - Setor de Educação da Universidade Federal do Paraná, Curitiba, 2013. 
MACHADO, V; HOLANDA, V.B. Diretrizes e Modelo Conceitual de Custo para o Setor Público a Partir da Experiência no Governo Federal do Brasil. RAP, Rio de Janeiro, v.44, no 4. p. 791-820, jul./ago. 2010.

MEEK, V. L. Policy change in higher education: intended and unintended outcomes. Higher Education, v. 21, (4) p. 451-459, jun. 1991.

MELO, J. C. C. B. S; MEZA, L. A; GOMES, E. G; NETO, L. B. Curso de Análise de envoltória de dados. XXXVII Simpósio Brasileiro de Pesquisa Operacional, Gramado/RS. p. 20520-20547, set., 2005.

MOTTA, P. R. Avaliação da Administração Pública: Eficiência, Eficácia e Efetividade. São Paulo: FUNDAP, 1990.

REED, R. J.; HURD, B. A value beyond money? Assessing the impact of equity scholarships: from access to success. Studies in Higher Education, v. 41, $\mathrm{n}^{\circ} .7$, p.1236-1250, 2016.

RICHARDSON, R. J. Pesquisa social: métodos e técnicas. 3. ed. São Paulo: Atlas. 1999.

SANTOS, C. P. C.; MARAFON, N. M. A Política de Assistência Estudantil na Universidade Pública Brasileira: desafios para o Serviço Social. Textos \& Contextos, v. 15, n 2, p. 408-422, ago/dez, 2016.

SCHWARTZMAN, S. Funções e metodologias de avaliação do ensino superior. In: Avaliação da Universidade: proposta e perspectivas. Brasília: Conselho de Reitores das Universidades Brasileiras, 1988. p.21-46. (Estudos e Debates, 14)

SLOMSKI, V. Controladoria e governança na gestão pública. São Paulo: Atlas, 2005.

ZYLBERSZTAJN, D.; SZTAJN, R. Direito e Economia: análise do direito e das organizações. Rio de Janeiro: Elsevier, 2005.

CRISTIANE APARECIDA DA SILVA é mestranda do Programa de Pós-Graduação em Contabilidade da Universidade Federal de Santa Catarina-UFSC. Contadora da Universidade Federal da Grande Dourados-UFGD. E-mail: cristianedasilva@ ufgd.edu.br 
FABRICIA SILVA DA ROSA é doutora em Engenharia da Produção com PósDoutorado em Contabilidade. Professora do Programa de Pós-Graduação em Contabilidade da Universidade Federal de Santa Catarina-UFSC. E-mail: fabricia. rosa@ufsc.br

ERNESTO FERNANDO RODRIGUES VICENTE é Doutor em Administração com Pós-Doutorado - Universidade do Porto, FEP-UP, Portugal. Professor do Programa de Pós-Graduação em Contabilidade da Universidade Federal de Santa Catarina-UFSC. E-mail: ernesto.vicente@ufsc.br

MARCIELE ANZILAGO é Doutoranda do Programa de Pós-Graduação em Contabilidade da Universidade Federal de Santa Catarina-UFSC. E-mail: marcianzilago@gmail.com

Recebido em agosto de 2018 Aprovado em novembro de 2018 